\title{
MICROBIOTA
}

\section{Tolerating gluten-a role for gut microbiota in celiac disease?}

Celiac disease affects individuals of all ages. Increasingly, the disease is diagnosed in adulthood, despite ingestion and tolerance of gluten for a number of years. New research by Alessio Fasano and colleagues makes tentative steps in understanding the cause of this change in immune tolerance to gluten. Could the gut microbiota have a role?

Taking a combined genomic and metabolomic approach, the investigators tried to decipher the potential effects of longitudinal changes in the gut microbiota and timing of gluten introduction on onset of celiac disease autoimmunity.

The researchers enrolled 34 infants who were genetically at risk of celiac disease (positive for HLA-DQ2 and/or HLA-DQ8) to the study and randomly assigned them to two groups: a glutenfree diet from age 6-12 months (delayed exposure) and a gluten-containing diet from 6 months (early exposure). All infants were breastfed until 6 months and resumed a normal diet from 12 months; 13 infants in each group completed the protocol.

The infants' gut microbiota was analyzed at time points from 7-days-old to 24 months of age. Contrary to existing data, the researchers found that children at risk of celiac disease had decreased abundance of Bacterioidetes. Moreover, "their microbiome was unstable and immature," adds Fasano.

Analysis of fecal metabolites from selected infants revealed no major differences between the dietary intervention groups. However, retrospective analysis of samples from an infant who later had a confirmed diagnosis of celiac disease revealed high levels of lactate before the onset of celiac disease autoantibodies, which Fasano believes might be useful in predicting the switch from tolerance to immune response.

These early findings will need to be replicated in larger studies; the authors are already aiming to recruit 800 infants to a multicenter study in the USA to confirm their data using a similar microbiomemetabolome analytical approach.

Katrina Ray

Original article Selitto, M. et al. Proof of concept of microbiome-metabolome analysis and delayed gluten exposure on celiac disease autoimmunity in genetically atrisk infants. PLoS ONE 7, e33387 (2012)

Further reading Volta, U. \& De Giorgio, R. New understanding of gluten sensitivity. Nat. Rev. Gastroenterol. Hepatol. doi:10.1038/nrgastro.2012.15
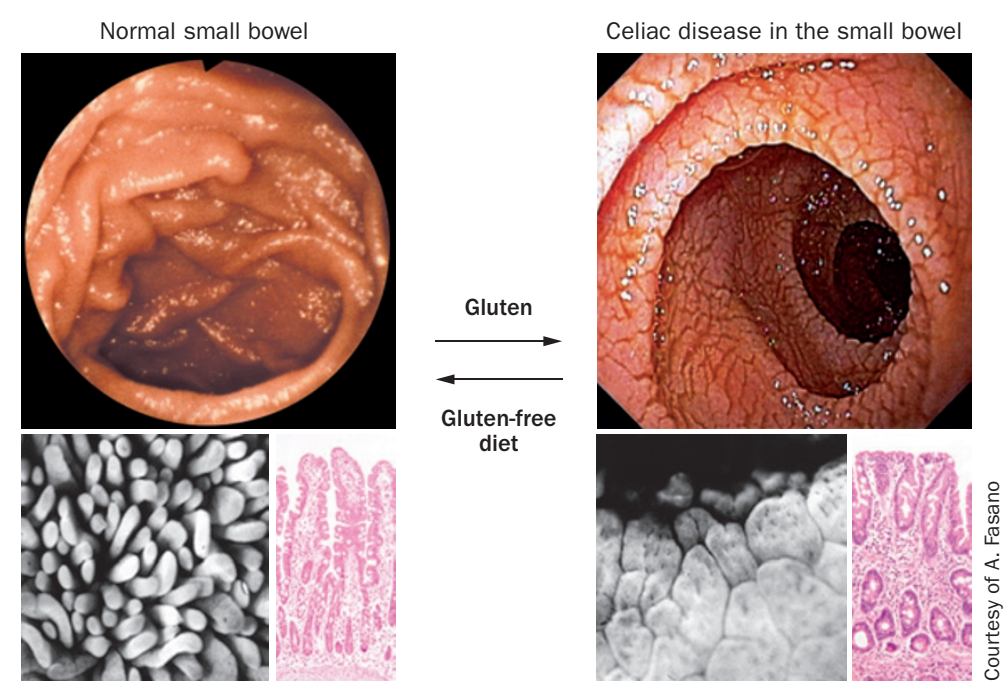